\title{
Quantum Interference and Spin Filtering Effects in Photo-responsive Endoperoxide Based Single Molecular Device
}

\author{
Ashima Bajaj, Rishu Khurana, and Md. Ehesan Ali* \\ Institute of Nano Science and Technology, Sector-81, Mohali, Punjab-140306, India \\ E-mail: ehesan.ali@inst.ac.in
}

\begin{abstract}
The development of stimuli responsive systems that can switch between two distinct spin states under the application of an external stimuli has always remained an illusory challenge. Here, we report a stimuli-based spin filter by utilizing photo-responsive endoperoxide (EPO) based single molecule device. The photo-irradiation on EPO triggers the homolytic cleavage of the peroxide $\mathrm{O}-\mathrm{O}$ bond generating diradical intermediate centered on two O-atoms which facilitates high spin filtering efficiency when placed between gold electrodes. The broken conjugated scenario due to peroxide bridge of EPO hinders the propagation of de Broglie wave across the molecular skeleton. While the diradical intermediate of EPO yields high conductance for one of the spin configuration. The transmission characteristics of various photoproducts along the photochemical reaction pathway of EPO are also investigated using density functional theory in combination with non-equilibrium Green's function (NEGF-DFT) technique. We demonstrate the key role played by Quantum Interference (QI) effects in dramatic modulation of conductance arising due to different degree of conjugation along the reaction pathway of EPO.
\end{abstract}




\section{Introduction}

The use of organic materials as a spin selective transport material for spintronics applications has raised broad interests. ${ }^{1}$ The weak spin-orbit coupling and long-spin relaxation times of organic materials as compared to transition metal complexes makes organic semiconductors appealing candidates for spintronics applications. The use of persistent organic radicals for inciting the spin polarized current through a single molecule connected to source and drain electrodes is well understood. ${ }^{2-4}$ However, instead of the use of permanent magnetic subunits, the more practical route is the development of stimuli-responsive systems that can switch between two distinct spin states once triggered with external stimuli such as light, mechanical force, electric field, magnetic field and chemical stimuli. ${ }^{5}$ The utilization of electric field to directly manipulate spin electrons was highlighted by Gaudenzi et al. by placing a PTM-based radical in a 3-terminal device, wherein the added electron by means of gate electrode changes the spin state from singlet to doublet. ${ }^{6}$ Hayakawa et al. reported magnetic field induced high magnetoresistance effects in stable TEMPO-OPE based single radical molecular junction. ${ }^{7}$ Apart from the electrical and magnetic stimuli responsive spin systems, van der Zant et al. also developed an approach to switch the spin state of central metal ion upon mechanical pulling of source and drain electrodes. ${ }^{8}$

Light has been widely used as the most promising and successful external control stimuli for designing single molecule electrical switches whose conductance can be reversibly turned on and off. In this context, many photochromic molecules including azobenzene, diarylethene, stilbene, spiropyran have been utilized as single molecule electrical switches since they can be reversibly switched between two stable structures upon light irradiation. ${ }^{9,10}$ However, for molecular spintronics, a long standing quest is the evolution of the light-responsive molecular spin system in which intramolecular spin alignment can be controlled by the action of light. The integration of such photoactive units in solid state devices could potentially yield efficient ultrafast manipulation of a spintronic device. Meanwhile, various photoactive 
diradicals have been studied by using photochromic molecule as a spin coupler between two magnetic units resulting in photoinduced magnetic crossover. ${ }^{11-13}$ Teki and co-workers investigated the spin alignment process within a $\pi$-conjugated radical built from a persistent oxo-verdazyl radical attached to anthracene unit which act as spin polarized electron donor upon photoexcitation. ${ }^{14}$ However, owing to the highly reactive nature of the radical species, the realization of such photomagnetic switches in device applications with the persistent spin sources is a challenging task and has not been accomplished yet. On the other hand, transient spin carriers could be of utmost importance here to achieve ultrafast control of molecular spintronic properties. In this context, recently Suda et al. developed a light driven reconfigurable spin filter employing chiral-induced spin selectivity effect. ${ }^{15}$

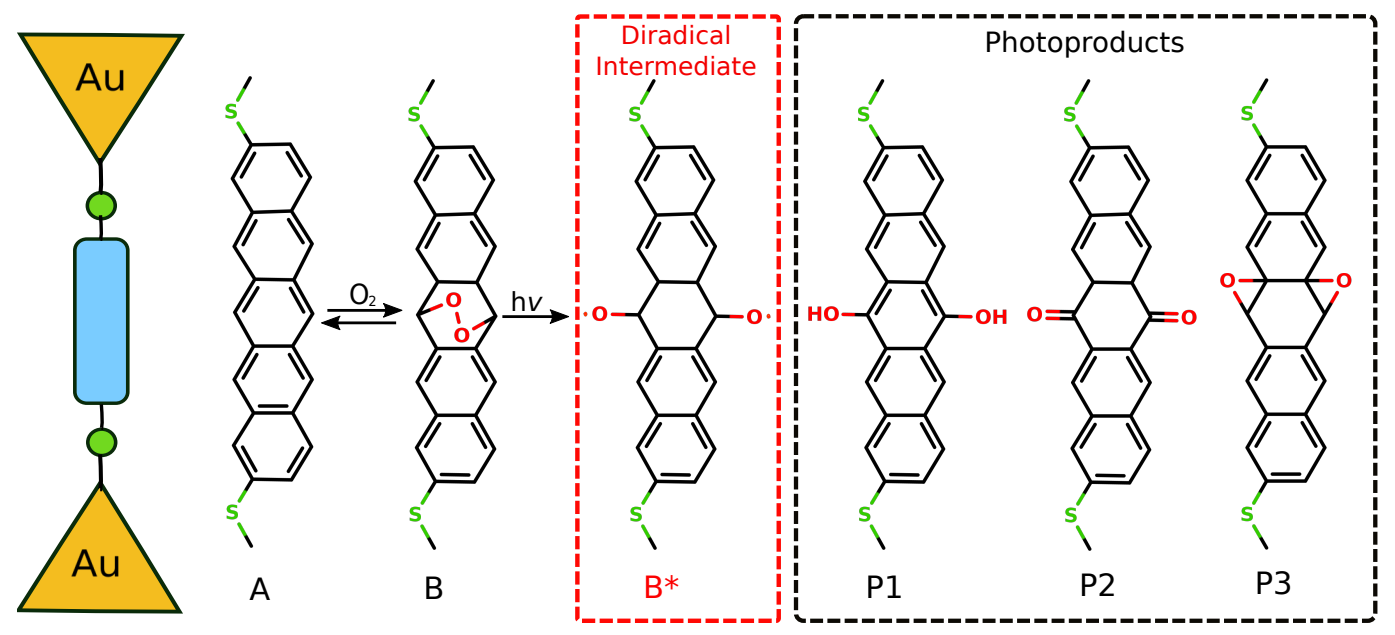

Figure 1: Junction schematic and molecular structures of the intermediates involved in the photochemical reaction of endoperoxide (EPO). The parent pentacene (A) in the presence of molecular oxygen yields pent-EPO (B). The photoexcited diradical intermediate state $\left(\mathrm{B}^{*}\right)$ shown in red enclosure decomposes into various possible photoproducts including dihydroxypentacene $(\mathrm{P} 1)$, pentaquinone $(\mathrm{P} 2)$ and diepoxypentacene (P3) rearrangement products shown in black enclosure.

Here, in this work, we propose an optically driven spin filter employing endoperoxide (EPOs) based molecular junctions where the molecule is coupled to gold electrodes. The photochemistry of EPOs has always remained illusive and the subject of extensive experimental attention in the past. ${ }^{16,17}$ As illustrated in Fig. 1, when exposed to air and light, acenes undergo a reaction with aerated oxygen to give EPOs with $\mathrm{O}-\mathrm{O}$ single bond as a peroxidic bridge 
in the central ring. Upon further photoexcitation, the homolytic cleavage of the peroxide

O-O bond takes place producing diradical intermediate along the reaction pathway. ${ }^{18}$ The generated diradical intermediate eventually decomposes into quinones, hyrdoxyquinones or diepoxides as rearranged photoproducts. ${ }^{19}$ The likely existence of a diradical intermediate reachable by the photoactivation of EPO ground state is unambiguously reported and has been exploited for numerous photobiological processes including selective destruction of cancer cells and activation of anti-malarial drugs. ${ }^{20-22}$ Besides this, EPOs also stand out as the chemical source of singlet oxygen storing systems where it enters another competing cycloreversion pathway to parent acenes via the cleavage of C-O bond. ${ }^{23}$ Despite their obvious appeal as spin polarized candidates, EPOs have seldom been used in molecular spintronics. Here, we undertake a theoretical study, demonstrating that the spin selective transmission mediated by the diradical intermediate of EPO can be successfully employed in device applications to induce transient spin polarization. The spin dependent electron transport signatures in molecular junctions have been investigated using the non-equilibrium Green's function approach combined with density functional theory (NEGF-DFT). We analyze how the structural reorganization and the changes in molecular electron density distribution by optical excitation affects the transport properties in junctions with a primary focus on the involved diradical intermediate. Furthermore, the results demonstrate the key role played by quantum interference (QI) effects in controlling the conductance throughout the entire photochemical reaction pathway.

\section{Computational Details}

Firstly, the geometries of isolated molecules involved in the photochemical reaction pathway, that is, A, B , B*, P1, P2 and P3 (shown in Fig. 1) are optimized including the two S-H bonds at both the ends using B3LYP/def2-TZVP method in ORCA. ${ }^{24}$ As depicted in Fig. 2, the optimized molecules are then sandwiched between two gold $\mathrm{Au}(111)$ electrodes after 
the dissociation of terminal hydrogen atoms from the thiol anchoring groups to form single molecule junctions. The molecular junctions are divided into three parts: the central region and semi-infinite left and right electrodes. The two semi-infinite Au electrodes are simulated by $5 \times 5$ unit cell with three layers in z-direction and periodic boundary conditions imposed in xy plane. The central region includes the molecule and parts of the left and right electrodes, which contains the four-atom Au-tetrahedron to form conical junction and extra one layer of Au-atoms on each side to replicate with the bulk electrodes. The central region is then optimized again with a maximum residual force of $0.02 \mathrm{eV} / \AA$ using PBE exchange correlation functional as implemented in SIESTA code. ${ }^{25}$ In this step, only molecule is allowed to relax within the junction, while all the Au atoms are kept fixed in their bulk lattice positions. In the relaxed junction, Au-S distance is found to vary from 2.35 to $2.40 \AA$.

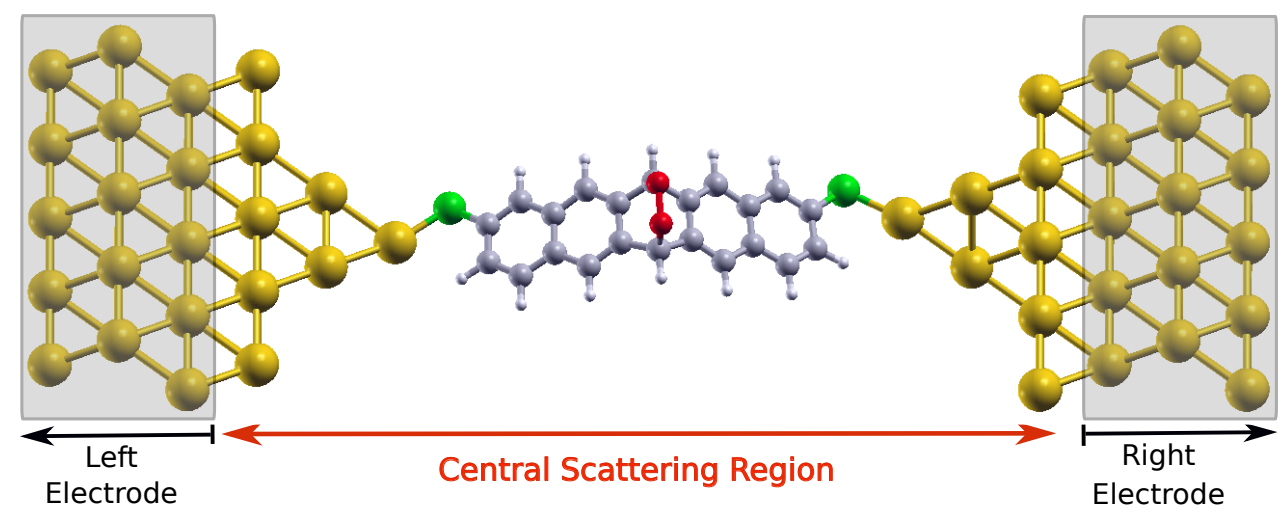

Figure 2: Device set up of Au-Molecule-Au junction used in electron transport calculations illustrating pent-EPO molecule placed between two $\mathrm{Au}(111)$ electrodes via sulphur anchoring atoms. Central scattering region consists of central molecule and 4-atom conical gold junction with an additional $5 \times 5$ layer of $\mathrm{Au}$ atoms.

The spin dependent electron transport properties are calculated using TranSIESTA code ${ }^{26}$ adopting density functional theory (DFT) based non-equilibrium Green's function (NEGF) method. ${ }^{27,28}$ Norm-conserving pseudopotentials are used to describe the core electrons. ${ }^{29}$ DZP basis set is used for molecular atoms while SZP basis set is used for Au-atoms. A mesh cutoff of 300 Ry is used with Brillouin zone sampling using $3 \times 3 \times 10$ k-points in $\mathrm{x}, \mathrm{y}$ and $\mathrm{z}$ directions. The electron transport is along z-direction. The transmission function is 
computed in the spirit of Landauer ${ }^{30}$ formalism using equation

$$
T_{\sigma}(E)=\operatorname{Tr}\left(\Gamma_{R} G \Gamma_{L} G^{\dagger}\right)_{\sigma}
$$

where $\sigma$ represents the spin up/down electrons. $G / G^{\dagger}$ indicates the retarted/advanced Green's function of the central region given by

$$
G(E)=\left(E S-H-\Sigma_{L}-\Sigma_{R}\right)^{-1}
$$

where $\mathrm{H}$ is the Hamiltonian and $\mathrm{S}$ is the overlap matrix. $\Gamma_{L} / \Gamma_{R}$ describes the level broadening due to coupling between the central region and Left/Right electrode given by

$$
\Gamma_{L, R}=\iota\left(\Sigma_{L, R}-\Sigma_{L, R}^{\dagger}\right)
$$

where $\Sigma_{L, R}$ are the self-energies associated with this coupling. The zero bias conductance is then obtained as

$$
G=G_{\circ} T\left(E_{F}\right)
$$

where $G_{\circ}=2 e^{2} / \mathrm{h}$ is the quantum of conductance. The spin filtering efficiency (SFE) at the Fermi level is calculated by using equation:

$$
S F E=\frac{\left|T_{\alpha}\left(E_{F}\right)-T_{\beta}\left(E_{F}\right)\right|}{T_{\alpha}\left(E_{F}\right)+T_{\beta}\left(E_{F}\right)}
$$

\section{Results and Discussion}

The gas phase geometries of the isolated molecules involved in the photochemical pathway of EPOs (shown in Fig. 1) are optimized at B3LYP/def2-TZVP level. The geometric comparison reveals that for $\mathrm{B}$ and $\mathrm{B}^{*}$, besides the $\mathrm{O}-\mathrm{O}$ cleavage $\left(d_{\mathrm{o}-\mathrm{o}}\right)$, butterfly bending of pentacene moiety defined as the angle $(\phi)$ between the two flaps bisected by mirror plane containing 
the O-O bridge (shown in Fig. 3a) serve as another degree of freedom characterizing O-O homolysis products. In equilibrium geometry, pent-EPO (B) is characterized by a distance $d_{\mathrm{o}-\mathrm{o}}=1.47 \AA$ and angle $\phi=126.49^{\circ}$.
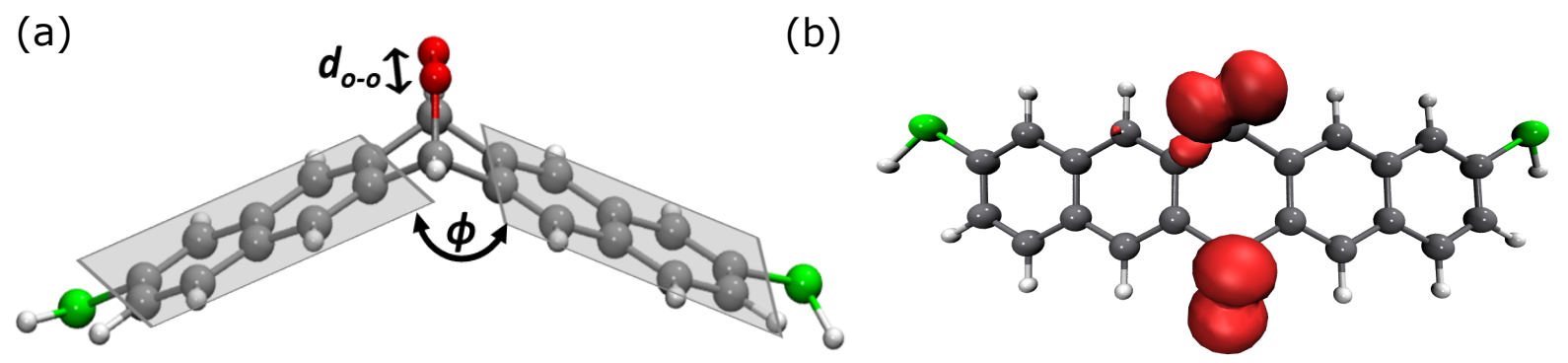

Figure 3: (a) Molecular geometry of pent-EPO (B) optimized at B3LYP/def2-TZVP level where $d_{\mathrm{o}-\mathrm{o}}$ is the distance between two O-atoms and $\phi$ is the angle between two planes bisected by the mirror plane containing O-O peroxide bridge. (b) Spin density plot of diradical intermediate $\left(\mathrm{B}^{*}\right)$ obtained using B3LYP/def2-TZVP method illustrating spin density localized on two O-atoms.

For EPOs, it has been demonstrated by several reports that upon irradiation, an electron is excited from $\pi^{\star}$ oo to antibonding $\sigma^{\star}$ oo which corresponds to the homolytic cleavage of O-O bond leading to first excited state. ${ }^{23,31,32}$ However, excitation to higher excited states, that is, $\mathrm{S}_{\mathrm{n}}(\mathrm{n} \geq 2)$ leads to local and charge transfer excitations corresponding to the generation of singlet molecular oxygen. ${ }^{33}$ Using MS-CASPT2/CASSCF methods, Mollenhauer et al. computed the photochemical pathway leading to O-O bond dissociation showing that the photo-dissociation process is governed by the degeneracy of four singlet plus four triplet states and predicted the diradical intermediate geometry corresponding to 8 -fold degeneracy. ${ }^{34}$ Since the determination of electronically excited states of EPO is not the prime focus of this work, we obtained the ground state structure of diradical species $\left(\mathrm{B}^{*}\right)$ that can function as the electronically excited diradical intermediate of pent-EPO (B) by the homolytic scission of peroxide $\mathrm{O}-\mathrm{O}$ bond and found it to be adiabatically located at $0.92 \mathrm{eV}$ from pent-EPO (B) ground state. The structure corresponding to diradical intermediate $\left(\mathrm{B}^{*}\right)$ shows a larger distance of $d_{\mathrm{o}-\mathrm{o}}=3.54 \AA$ and more planar pentacene moiety as compared to pent-EPO (B) with $\phi=156.39^{\circ}$. This is in fair agreement to the excited state geometry predicted by 
Mollenhauer et al. using SA-CASSCF methods for anthracene-EPO. ${ }^{34}$ Apart from this, broken symmetry calculations on B* state at B3LYP/def2-TZVP level reveals singlet triplet energy gap of $73.05 \mathrm{~cm}^{-1}$ with a ferromagnetic ground state and $<S^{2}>_{B S}$ value of 1.00 indicating ideal singlet-state diradical configuration of $\mathrm{B}^{*}$. The spin density plot of $\mathrm{B}^{*}$ shown in Fig. 3b also illustrates that homolytic O-O scission in EPO breaks the spin symmetry and yields ferromagnetic ordering with the spin density localized on the two O-atoms. The intermediate diradical centered on O-atoms $\left(\mathrm{B}^{*}\right)$ then eventually decomposes to diverse photoproducts such as quinones, hydroxyquinones or diepoxides depending upon the solvent conditions and substitutents. Here, we will first discuss the evolution of transmission characteristics for EPO $(\mathrm{B})$ and its diradical intermediate $\left(\mathrm{B}^{*}\right)$ starting from the parent pentacene $(\mathrm{A})$. In the subsequent discussion, we will discuss how QI effects can be harnessed to optimize the response of EPO based junction through various possible photochemical pathways.

\subsection{Spin Resolved Transport in EPO and Diradical Intermediate}

The spin-resolved transmission spectra of parent pentacene (A), pent-EPO (B) and its diradical intermediate $\left(\mathrm{B}^{*}\right)$ is shown in Fig 4 . For parent pentacene, molecule has a conjugated form and completely planar geometry. While for pent-EPO, the orbital hybridization of edge carbon sites of the central benzene ring changes from $s p^{2}$ to $s p^{3}$ and the molecule loses conjugation in the central benzene ring. The broken conjugation in the central ring results in butterfly bending of EPO geometry exhibiting an angle $\phi=126.49^{\circ}$ as already discussed in previous section and shown in Fig. 3. The hybridization of molecule with the gold electrodes also preserves similar geometrical features for both pentacene as well as pent-EPO once inserted in molecular junction. Similarly, for the diradical intermediate, the cleavage of O-O bond results in planar pentacene moiety with $\phi \approx 150^{\circ}$ for both isolated molecule as well

as in junction. However, the distance between two O-atoms in $\mathrm{B}^{*}$ changes from $3.54 \AA$ for isolated molecule to $2.18 \AA$ in molecular junction.

The transmission spectra reveals that for parent pentacene, HOMO and LUMO resonance 
peaks are located at around -0.20 and $0.62 \mathrm{eV}$ below and above Fermi energy respectively and yields a HOMO-LUMO gap of $0.82 \mathrm{eV}$ in junction with a conductance of the order of $10^{-3}$ at Fermi level. This is accompanied by a sharp dip of the order of $10^{-5}$ located just above the Fermi energy arising due to destructive quantum interference (DQI) between the de-Broglie waves traversing along different pathways. The DQI effects in meta connected pentacene is discussed in details in our previous work ${ }^{4}$ which is in well agreement with several experimental reports for meta connected benzene. ${ }^{35,36}$ In contrast, the transmission spectra of pent-EPO (shown in middle panel of Fig. 4), reveals the alleviation of sharp DQI dips with two broad peaks corresponding to HOMO and LUMO resonances located at -0.60 and $1.48 \mathrm{eV}$ respectively. However, due to larger HOMO-LUMO gap of $2.08 \mathrm{eV}$, conductance is deliberately reduced to the order of $10^{-4}$ at Fermi level. The electronic structure calculations for isolated molecule at B3LYP/def2-TZVP level also reveals that HOMO-LUMO gap increases from 2.16 to $4.12 \mathrm{eV}$ when going from fully conjugated pentacene to broken conjugated EPO (see Fig. S1 of SI). Similar effects have been previously observed in several experimental and theoretical reports demonstrating the transport characteristics of molecules with different degrees of conjugation. ${ }^{37-39}$ 


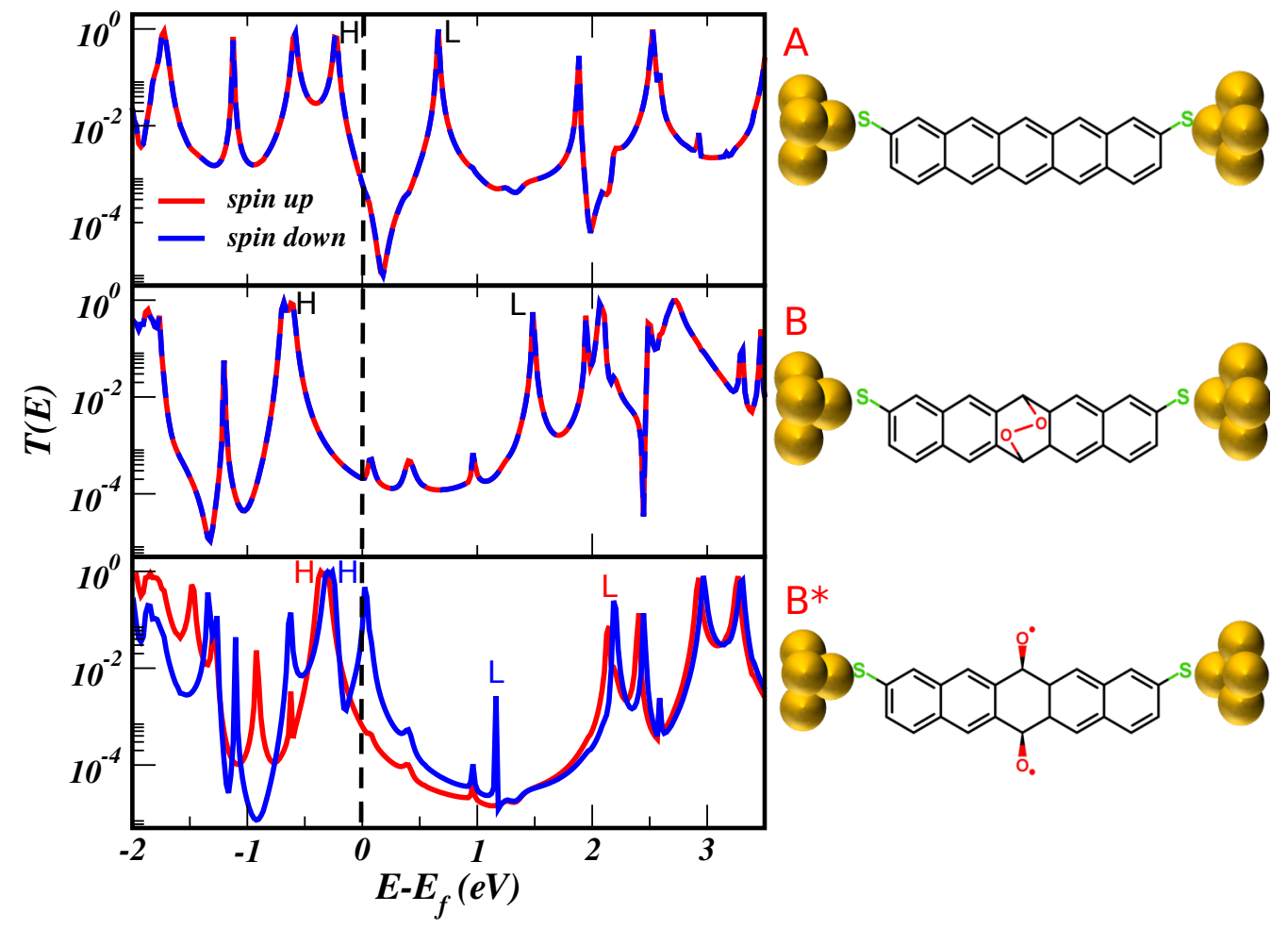

Figure 4: Spin-resolved transmission spectra of parent pentacene (A), pent-EPO (B) and its diradical intermediate $\left(\mathrm{B}^{*}\right)$. The corresponding molecules held between two meta connected $\mathrm{Au}$ electrodes are illustrated alongwith. The spin-up and spin-down transmission is denoted by red and blue colors respectively. Vertical dotted line denotes the position of Fermi energy.

Further for pentacene and pent-EPO, owing to spin degenerate levels, spin up transmission replicates with that of spin down displaying absence of spin polarization and zero SFE. On the other hand, the transmission spectra of diradical intermediate obtained by O-O homolytic scission (shown in bottom panel of Fig. 4) displays distinct $\alpha$ and $\beta$ transmission curves especially around the Fermi level from -2.0 to $1.0 \mathrm{eV}$. The spin up transmission of $\mathrm{B}^{*}$ displays two resonances at -0.30 and $2.15 \mathrm{eV}$ below and above Fermi energy respectively with a wider HOMO-LUMO gap of $2.45 \mathrm{eV}$. Akin to EPO, the spin up conductance is severely suppressed to the order of $10^{-4}$. On the other hand, the spin down transmission spectra reveals three dominant peaks with two of them residing below and above Fermi energy at -0.20 and 1.16 $\mathrm{eV}$ respectively and one peak anomalously centered at Fermi energy. More interestingly, the anomalous peak located at the Fermi energy of spin down transmission spectra yields higher 
conductance of the order of $10^{-2}$ and results in perfect spin filtering behaviour with high SFE of $97.8 \%$.

To get a deeper understanding of the different features observed in the spin-resolved transmission spectra of $\mathrm{B}^{*}$, density of states projected (PDOS) onto the $\mathrm{C}(2 \mathrm{p})$ and $\mathrm{O}(2 \mathrm{p})$ atomic orbitals is shown in Fig. 5. Here, the quest arises that whether the anomalous spin down peak at the Fermi energy is a resonance peak associated with a molecular channel or an additional state induced as a result of orbital hybridization between molecule and gold electrode known as hybrid interface states (HIS). To scrutinize this, we compared the total DOS of the isolated diradical B* (shown with grey color in left panel of Fig. 5) with the PDOS projected over the molecular atoms in junction (shown with grey color in right panel of Fig. 5). The total DOS of the isolated molecule reveals that the spin-down spectra exhibits two nearly degenerate peaks at $\sim 0.5 \mathrm{eV}$ above Fermi energy. The orbital resolved DOS further reveals that the two degenerate peaks arises from $\mathrm{p}_{\mathrm{z}}$-orbitals of two O-atoms. Further, the DOS projected over the p-orbitals of two individual O-atoms (Fig. S6 of SI) affirms that the two degenerate peaks individually corresponds to the $\mathrm{p}_{\mathrm{z}}$-orbitals of two different O-atoms wherein one peak shows a minor mixing with $\mathrm{p}_{\mathrm{x}}$-orbitals of one O-atom, while the other displays a mixing with $\mathrm{p}_{\mathrm{y}}$-orbitals of other O-atom. The spatial distribution of molecular orbitals of isolated molecule (shown in Fig. S4 of SI) further indicates one to one correspondence between the two degenerate peaks and closely aligned LUMO and LUMO+1 of the molecule localized on the different O-atoms. Moving to the molecular junction, the spin-resolved PDOS reveals a clear correspondence between the spin up and spin down peaks in transmission function (shown in bottom panel of Fig. 4) and the PDOS. However, in comparison to the DOS of the isolated molecule, the spin down PDOS spectrum in junction displays modified DOS near Fermi level wherein no any degenerate peaks are observed at $\sim 0.5 \mathrm{eV}$. Instead two additional peaks originating from p-orbitals of O-atoms appears at Fermi energy and $1.16 \mathrm{eV}$ above Fermi energy. At Fermi energy, there is a clear contribution arising from $\mathrm{p}_{\mathrm{z}}$ orbitals of radical centered O-atoms in the spin down PDOS, while there is 
no corresponding peak at Fermi energy for spin up channel. Apart from this, the spin down PDOS spectra displays another distinct green peak originating from $\mathrm{p}_{\mathrm{y}}$ orbitals of O-atoms at $1.16 \mathrm{eV}$ above Fermi energy. On the other hand, for spin up configuration, the PDOS is very much featureless in the energy window of 0 to $2 \mathrm{eV}$. This clearly indicates that spin bearing oxygen atoms plays a crucial role in inducing high spin filtering in the diradical intermediate of EPO. Further, from the PDOS comparison of isolated molecule and molecule in junction, one can intuitively understand that the orbital hybridization of the molecular orbitals with gold electrodes results in lifting of degeneracy of LUMO and LUMO+1 and yields two individual peaks located at Fermi energy and $1.16 \mathrm{eV}$ above it. Apart from this, the spin density distribution of $\mathrm{B}^{*}$ in isolated state and in molecular junction, computed at the same PBE level of theory, reveals ferromagnetic alignment of unpaired spins. However, for isolated $\mathrm{B}^{*}$, the unpaired spins reside in two orthogonal p-orbitals, while in junction the smaller distance between two O-atoms, that is, $d_{\mathrm{o}-\mathrm{o}}=2.18 \AA$, favors mixing of p-orbitals.
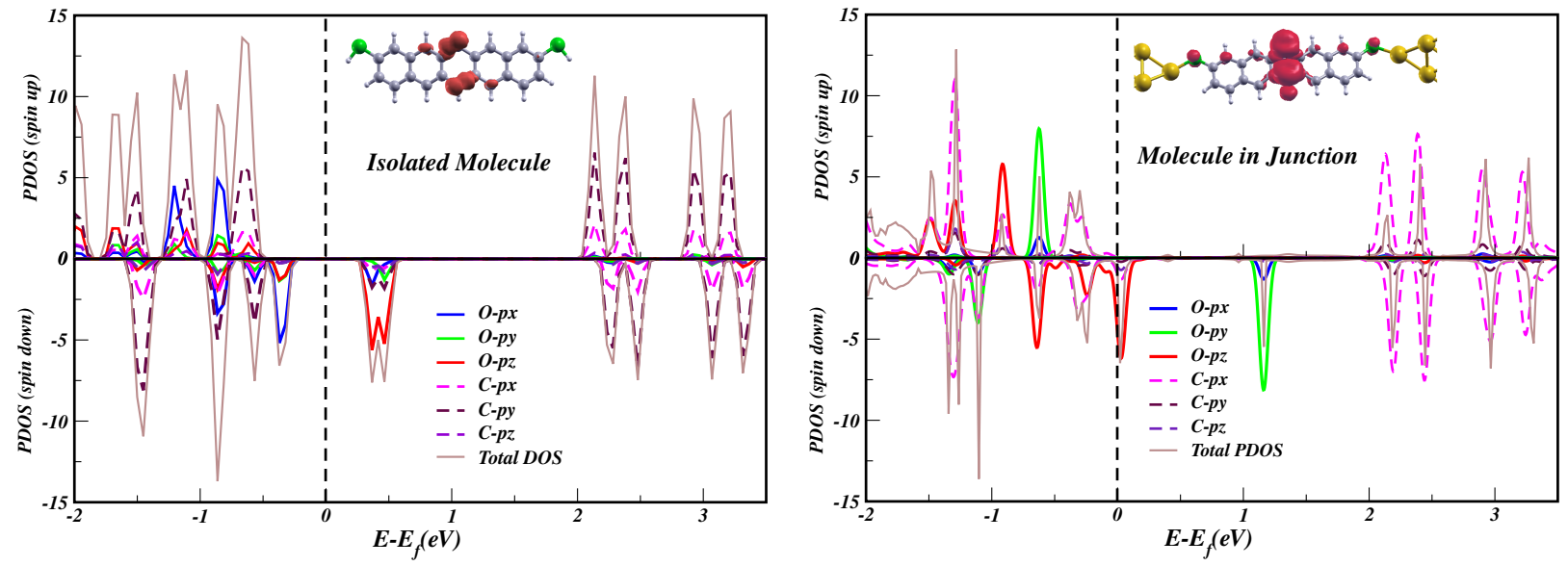

Figure 5: Projected density of states (PDOS) onto the $\mathrm{C}(2 \mathrm{p})$ and $\mathrm{O}(2 \mathrm{p})$ atomic orbitals of diradical intermediate $\left(\mathrm{B}^{*}\right)$ of EPO in isolated state (left panel) and in molecular junction (right panel). Grey color indicates total DOS for isolated B*, while in junction it corresponds to PDOS onto the scattering region. The PDOS comparison reveals lifting of degeneracy of LUMO and LUMO+1 upon placing $\mathrm{B}^{*}$ in junction. The spin density plots of $\mathrm{B}^{*}$ in isolated phase and in junction computed at PBE level is also shown in insets.

To further elucidate the nature of spin dependent channels of $\mathrm{B}^{*}$ in molecular junction, molecular projected self consistent Hamiltonian (MPSH) spectrum was computed. Formally, 
MPSH states represent modified molecular orbitals and electronic reorganization of energy levels in the presence of surrounding electrodes. The spatial distribution of MPSH states, shown in Fig. 6, reveals that for spin up configuration, there are two nearly degenerate states below the Fermi level at $\sim-0.30$ and $-0.37 \mathrm{eV}$ which are spatially separated with enhanced orbital density at the opposite sides. This clearly indicates that the cleavage of O-O peroxide bond breaks the spin-spatial symmetry and retains the ferromagnetic ordering of unpaired spins in junctions as well which can be clearly seen from the spin density plot of $\mathrm{B}^{*}$ in junction shown in inset of Fig. 5. Further, the MPSH spectra for spin down configuration reveals an interesting phenomena. As revealed from the PDOS analysis, the two degenerate unoccupied states (as observed in total DOS of isolated molecule) got lifted up in junction and results in two individual peaks located just near Fermi energy and $1.16 \mathrm{eV}$ above it. Here, the spatial distribution of MPSH states at $0.07 \mathrm{eV}$ and $1.16 \mathrm{eV}$ corresponding to the two peaks reveals that the one near Fermi energy at $0.07 \mathrm{eV}$ localized on the O-atoms displays an efficient coupling with gold electrodes. Thus provides a conducting channel for spin down electrons and yields higher conductance at Fermi level. While the other one located at $1.16 \mathrm{eV}$ shows inefficient coupling with electrodes and results in transmission peak of lesser amplitude as can be seen from the transmission spectra shown in bottom panel of Fig. 4. 

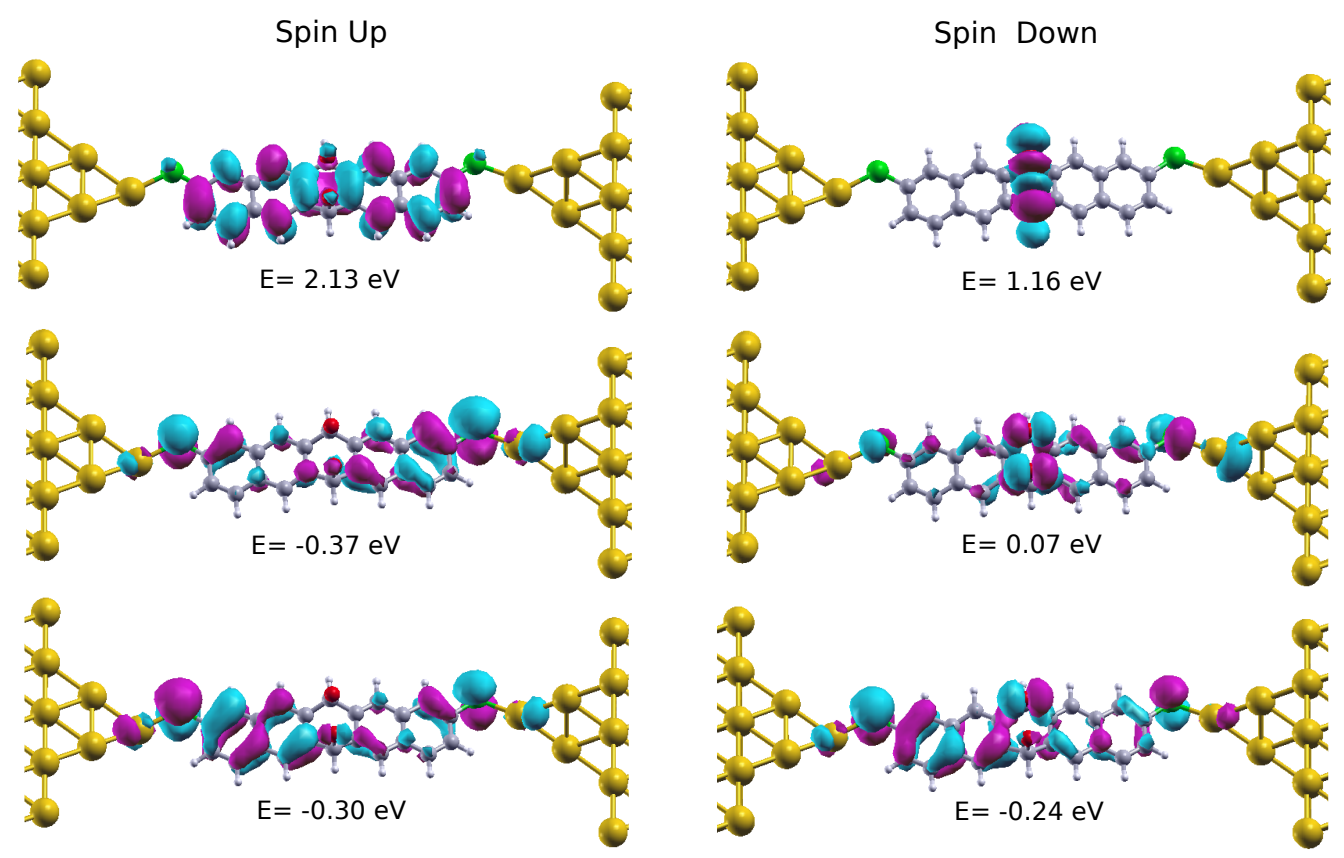

Figure 6: Spatial distribution of frontier MPSH states near Fermi energy of diradical intermediate $\left(\mathrm{B}^{*}\right)$ in junction. The spin up and spin down states are shown in left and right columns respectively.

Finally, Transmission Eigenchannel (EC) analysis for B and B* illustrated in Fig. 7 reveals that for pent-EPO, both Left EC and Right EC is vanished on the peroxide bridge. Thus indicating that the broken conjugation by the peroxide bridge introduces obstacles in the coherent charge propagation pathway by breaking the continuity of conjugation pathway between the electrodes. Hence, results in lower conductance as already inspected from the transmission spectra (shown in Fig. 4). For B*, spin up ECs replicates with that of of pent-EPO (B) with the ECs blocked by the peroxide bridge. In contrast, for spin down electrons, both the ECs are delocalized throughout the bridging molecule and extended on both the sides with larger amplitude than spin up ECs. Thus, indicating strong terminal coupling of spin down ECs. Further, spin down ECs plotted at larger isovalue of 0.05 (shown in inset) reveals that the spin down channel becomes conductive due to major contribution coming from radical centered $\mathrm{O}$-atoms and hence results in high conductance of spin down electrons and thus high SFE. 


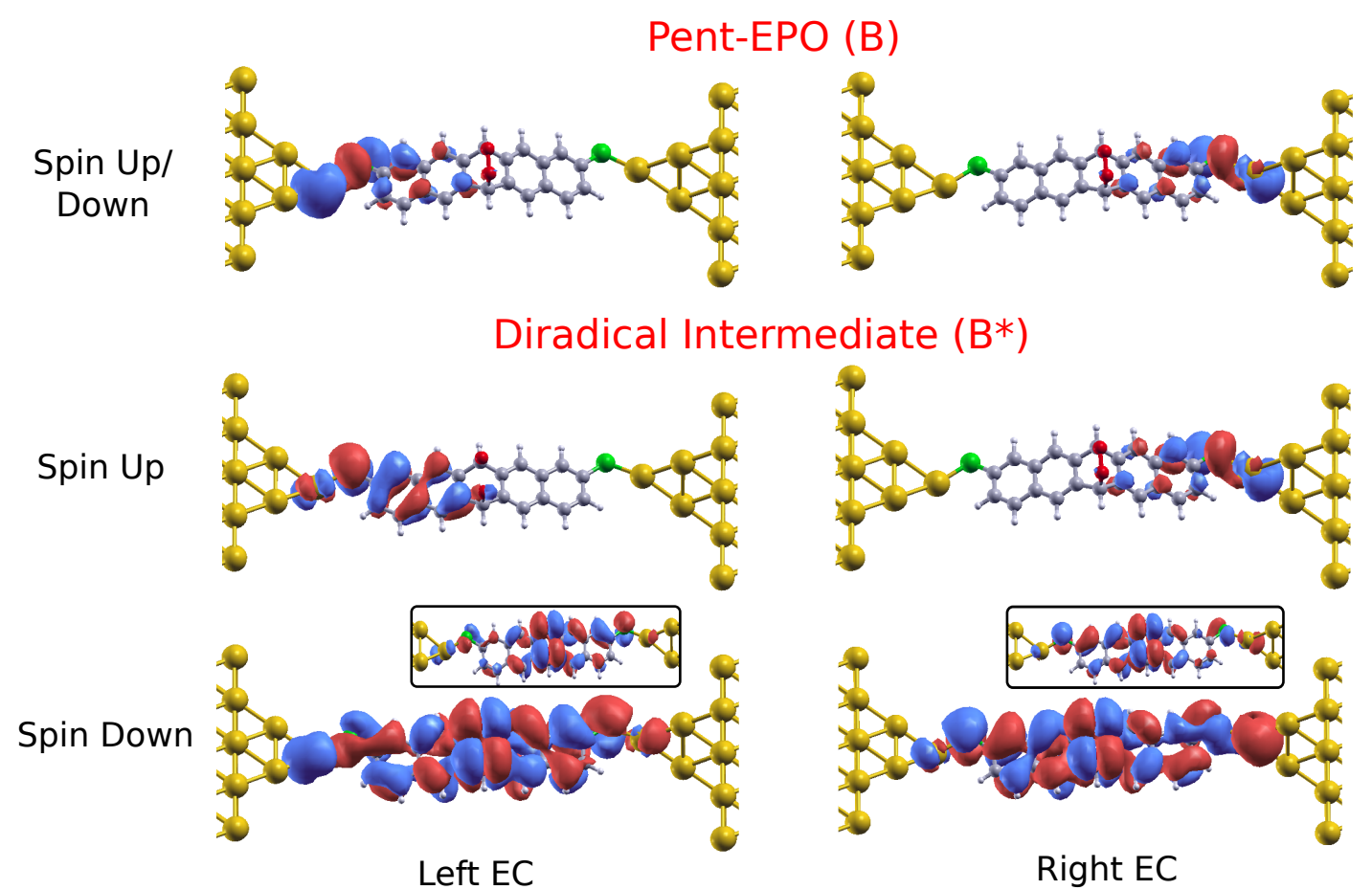

Figure 7: Transmission eigenchannels (EC) of pent-EPO (B) and diradical intermediate $\left(\mathrm{B}^{*}\right)$ (with an isovalue of 0.02). For EPO, both Left EC and Right EC is vanished on peroxide bridge. For B*, spin up ECs replicate with B, while spin down ECs are completely delocalized. To get a clear picture of the nature of spin down ECs, a larger isovalue of 0.05 is also used as shown in the black boxes.

\subsection{Role of Quantum Interference in EPO based device}

After gaining insights in to spin selective transport via the diradical intermediate of EPO, we turned out to explore quantum interference prospective in junctions containing EPO and the possible photoproducts (P1, P2 and P3) shown in Fig. 1. QI effects can lead to dramatic modulation and suppression of conductance in single molecule junctions by allowing or forbidding the de-Broglie waves from passing through a system. ${ }^{40}$ As for linearly $\pi$-conjugated molecules, it has been demonstrated that the molecules with meta connection to the electrodes often disrupts the conduction due to destructive QI (DQI). While the para connected analogs are predicted to be better conductors due to constructive QI (CQI). ${ }^{41,42}$ Furthermore, an illusory connection between cross conjugation and quantum interference has also been established in few of the prior reports demonstrating a conclusive evidence of DQI for para 
connected cross-conjugated quinone which is in contrast to linearly conjugated molecules. ${ }^{43-45}$ Here, across the photochemical reaction pathway of EPO, the molecule is subjected to different degrees of $\pi$-conjugation patterns. As for parent pentacene exhibits fully linearly conjugated geometry, while the formation of peroxide bridge results in broken conjugation scenario for pent-EPO (B) and its diradical intermediate $\left(\mathrm{B}^{*}\right)$. Apart from this, the various possible photoproducts includes dihydroxypentacene (labelled as P1) displays fully conjugated geometry, pentaquinone (labelled as P2) is a cross conjugated molecule and diepoxypentacene (labelled as P3) has a broken conjugated skeleton. Thus, we calculated transmission spectra for pent-EPO and its photo-products with both para and meta connections to gold electrodes as shown in purple and orange enclosures of Fig. 8a respectively. The corresponding transmission spectra is represented by purple and orange curves for para and meta connections respectively in Fig. 8b. In what follows, we investigate how the different $\pi$-conjugation patterns of the molecule across the photochemical pathway of EPO can affect the molecular conductance such that QI features can be toggled on and off by the external stimuli to realize a QI based molecular switch. 
(a)
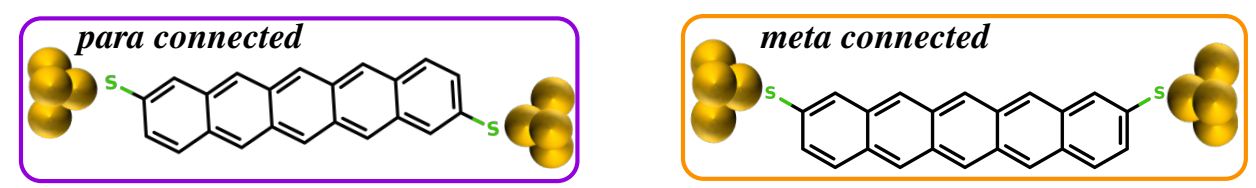

(b)

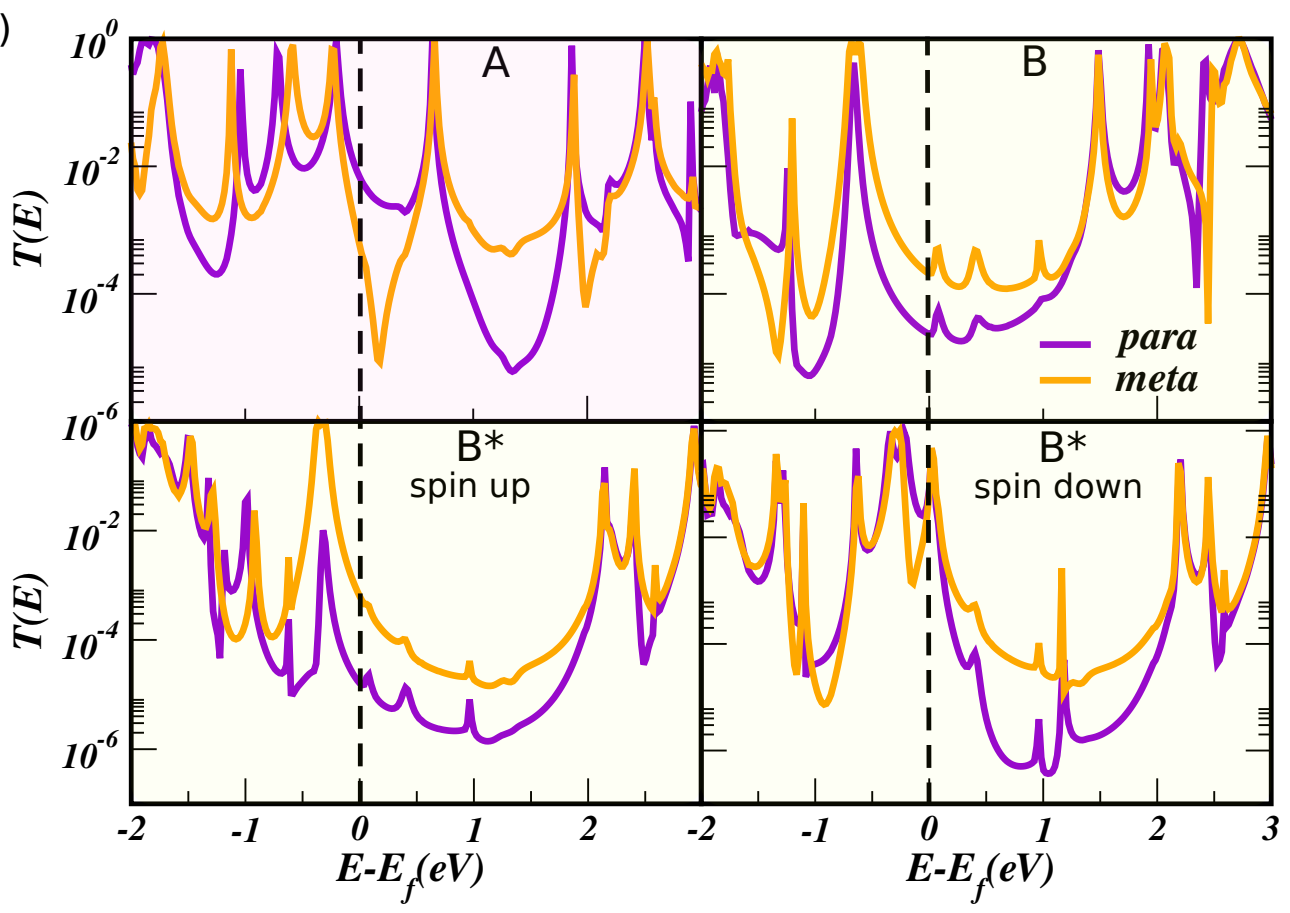

Figure 8: (a) Parent pentacene (A) held between two Au electrodes illustrating para and meta connections in purple and orange enclosures respectively. (b) Transmission spectra of pentacene (A), pent-EPO (B) and both spin configurations of diradical intermediate $\left(\mathrm{B}^{*}\right)$, where purple and orange curves corresponds to molecules with para and meta connections respectively. The vertical dotted line denotes the position of Fermi level. Pentacene has fully conjugated geometry while B and $\mathrm{B}^{*}$ exhibits broken conjugation in the central benzene ring.

As illustrated in Fig. 8b, for parent pentacene, QI manifests itself in the form of DQI for meta connected pentacene with a sharp dip of the order $10^{-5}$ near Fermi level, while para connected pentacene yields higher conductance of the order $10^{-3}$ due to CQI. The appearance of CQI and DQI in the respective para and meta connections is in complete agreement with previous theoretical and experimental studies on benzene and other polycyclic hydrocarbons. ${ }^{46-48}$ In complete contrast to this, for broken conjugated EPO and its diradical intermediate $\left(\mathrm{B}^{*}\right)$ in junction, the transmission spectra reveals no signatures of strong destructive interference dips in neither of the para and meta connections. To unveil whether the disappearance of strong DQI dips in EPO is associated with broken conjugated structure, 
we calculated transmission spectra for broken conjugated dihyrdropentacene (PH) obtained by replacing each radical centered oxygen atom of $\mathrm{B}^{*}$ with a hydrogen atom at the central benzene ring of pentacene. The transmission spectra of dihyrdropentacene (shown in Fig.S5 of SI) also reveals no any impression of DQI dips in neither of the para and meta connections. This clearly indicates that the broken conjugated structure of EPO abandons the presence of strong DQI dips. Thus, we conclusively postulate that the photo-oxygenation of pentacene to yield EPO results in switching off the QI features, thus opening another wider area of applications in which EPO based device can serve as a stimuli responsive QI based switch. Such a unique combination of stimuli-response and quantum interference has been rarely explored in the literature. ${ }^{49}$ Apart from this, the calculated conductance at the Fermi energy, tabulated in Table 1, reveals that for pent-EPO, the conductance of para connected junction is lesser than the meta connected one with a decreased para/meta conductance ratio of $10^{-1}$ as compared to pentacene with para connected pentacene being more conductive as compared to meta one. Analogous to EPO, the spin up configuration of B* also reveals decreased para/meta conductance ratio of $10^{-2}$. For spin-down, although the conductance values at Fermi energy are quite comparable for para and meta connection, but the transmission spectra shows lesser conductance of para connection than meta one over a wider energy range above Fermi energy. Notably, this behavior was not observed for broken conjugated dihyrdropentacene $(\mathrm{PH})$ for which the transmission spectra (shown in Fig.S5 of SI) and calculated conductance (tabulated in Table 1) reveals that the transmission is slightly lower for meta connected PH as compared to the para one around the Fermi energy. This clearly indicates that the reversal of para/meta conductance in $\mathrm{B}$ and $\mathrm{B}^{*}$ is not an absolute effect of broken conjugation, but could either be associated with the substituent effect in case of EPO or spin selective nature of its diradical intermediate. The similar decrease in para/meta conductance ratio was also observed by Herrmann et al. with the introduction of closed/open shell substituent acting as an additional site in the $\pi$ system. ${ }^{50}$ and is also in line with our recent finding where we exclusive demonstrated the reversal of para/meta conductance in the 
presence of persistent radical centers. ${ }^{4}$

Table 1: Calculated conductance $(\mathrm{G})$ in units of $\mathrm{G}_{0}$ for para and meta connected pent-EPO and the associated photo-products in junctions. The para/meta conductance ratio is also tabulated alongwith.

\begin{tabular}{cccc}
\hline \multirow{2}{*}{ Molecule } & \multicolumn{2}{c}{$\mathrm{G}\left(G_{\circ}\right)$} & \multirow{2}{*}{ para/meta } \\
\cline { 2 - 4 } & para & meta & \\
\hline Pentacene (A) & $9.65 \times 10^{-3}$ & $1.13 \times 10^{-3}$ & $8.53 \times 10^{0}$ \\
\hline Pent-EPO (B) & $2.34 \times 10^{-5}$ & $2.08 \times 10^{-4}$ & $1.12 \times 10^{-1}$ \\
\hline Intermediate (B*; spin up) & $1.61 \times 10^{-5}$ & $6.30 \times 10^{-4}$ & $2.50 \times 10^{-2}$ \\
Intermediate (B*; spin down) & $7.94 \times 10^{-2}$ & $6.75 \times 10^{-2}$ & $1.17 \times 10^{0}$ \\
\hline Pent-Hydroxy (P1) & $1.22 \times 10^{-2}$ & $4.73 \times 10^{-3}$ & $2.57 \times 10^{0}$ \\
\hline Pent-Quinone (P2) & $2.45 \times 10^{-7}$ & $2.75 \times 10^{-4}$ & $8.90 \times 10^{-4}$ \\
\hline Pent-Diepoxy (P3) & $2.91 \times 10^{-5}$ & $2.43 \times 10^{-5}$ & $1.19 \times 10^{0}$ \\
\hline Pent-Hydro & $3.03 \times 10^{-5}$ & $1.41 \times 10^{-5}$ & $2.14 \times 10^{0}$ \\
\hline
\end{tabular}

As a well known fact, the diradical intermediate generated by homolytic O-O cleavage of EPO has a shorter lifetime and it eventually decomposes to give diverse photoproducts depending upon the solvent conditions and substitutents. A complete overview of different photochemical reaction pathways identified for EPO has been enumeriated by several authors in the field. ${ }^{16,51}$ Fig. 9a illustrates the three most identified photoproducts of EPO, that is, dihyrdoxypentacene $(\mathrm{P} 1)$ obtained by hydrogen rearrangement reaction of $\mathrm{B}^{*}$, pentaquinone (P2) obtained by elimination of $\mathrm{H}_{2}$ from $\mathrm{B} *$ and diepoxypentacene (P3) which is identified as another dominant primary rearrangement product. The transmission spectra of possible photoproducts P1, P2 and P3 is shown in Fig. 9b. The first photoproduct, that is, fully conjugated dihyrdoxypentacene (P1) shows similar behavior as of pentacene bearing strong DQI dip for meta connected P1. The only difference is the slight shift in $\mathrm{T}(\mathrm{E})$ of $\mathrm{P} 1$ towards right as compared to pentacene, which is due to weak electrostatic gating effect of the oxygen atoms in P1. Another photo-product, pentaquinone (P2) bearing cross-conjugated molecular skeleton demonstrates DQI for para connected P2 and CQI for meta connectivity with para/meta conductance ratio of $10^{-4}$ which is in clear contrast to fully conjugated pentacene and dihyrdoxopentacene (P1). This generic feature of cross conjugated wires is well reported in several charge transport studies of cross conjugation versus linear conjugation where junctions 
containing an anthraquinone core are often cited as an example to successfully operate as an electrochemical switch. ${ }^{52-54}$ Lastly, the third photo-product, that is, diepoxypentacene (P3) with broken conjugation reveals almost indistinguishable $\mathrm{T}(\mathrm{E})$ of para and meta connections with a decreased conductance of the order of $10^{-5}$ as of pent-EPO. The observed QI features in EPO and photoproducts are further analysed with the aid of corresponding Transmission Eigenchannels (ECs) and are discussed in SI.

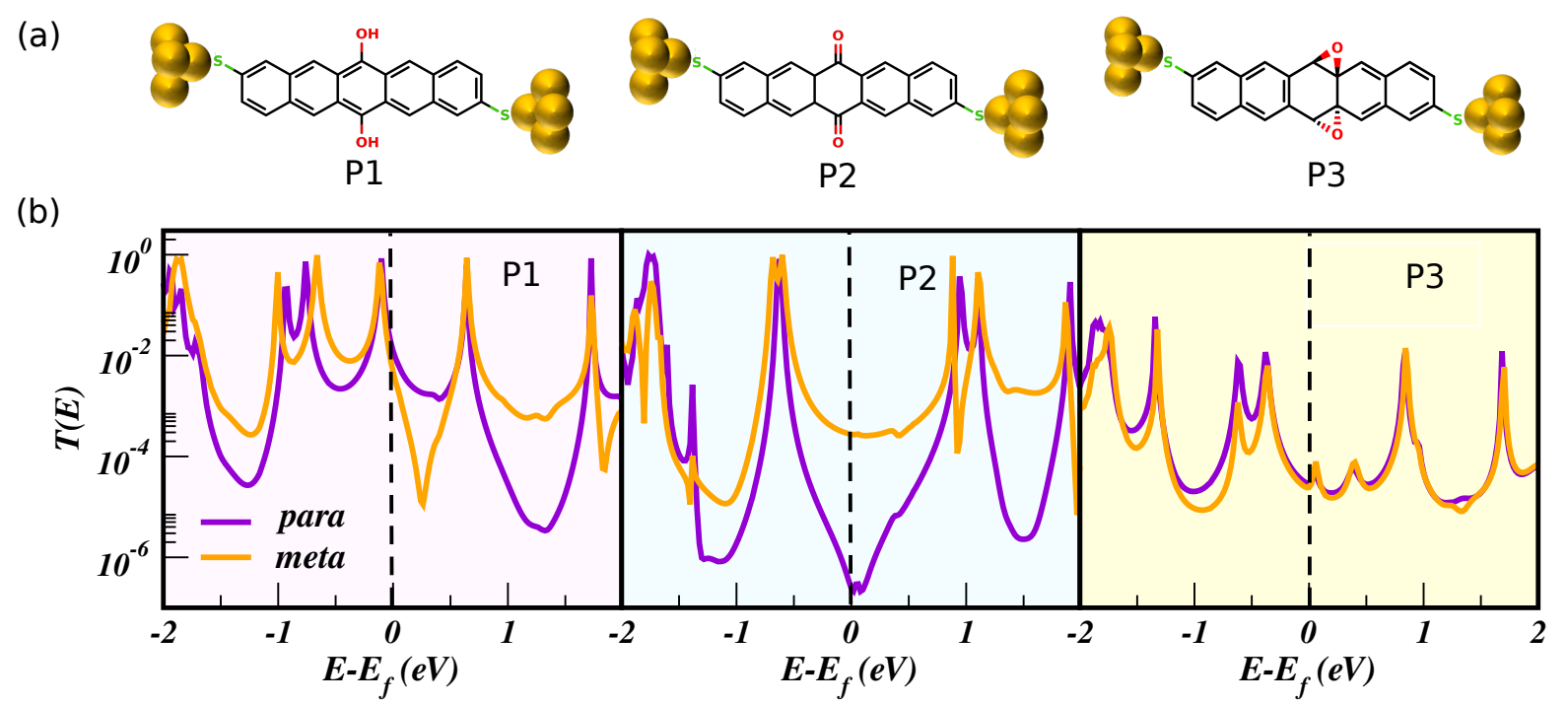

Figure 9: (a) Photoproducts of EPO namely dihyrdoxypentacene (P1), pentaquinone (P2) and diepoxypentacene (P3) in molecular junctions with para connections. The corresponding meta connections are shown in Fig. 1. (b) Calculated transmission spectra for para and meta connected P1, P2 and P3, where purple and orange curves corresponds to para and meta connections respectively. The vertical dotted line denotes the position of Fermi level.

Noteworthy, another interesting aspect of EPO is its dual mode of photochemistry where it has been vividly concluded that excitation to S1 state causes the homolytic O-O cleavage which eventually results in the formation of diradical intermediate. ${ }^{17}$ Whereas excitation to higher state $\left(S_{n}, n \geq 2\right)$ leads to cycloreversion reaction producing pentacene which again signifies the application of EPO based device as a photo-modulated reversible QI based electronic switch. Thus, to summarize, a significant variation in conductance can be realized starting from EPO to final photoproducts in both para and meta connections wherein QI features can be reversibly toggled on and off to realize a molecular switch. 


\section{Conclusions and Outlook}

In summary, the work demonstrates a dual functionality integrated into a EPO based device with nearly perfect spin filtering by its diradical intermediate and switchable QI effects. A high spin filtering efficiency of $97.8 \%$ is achieved for diradical intermediate of EPO wherein molecular channels for one spin configuration are blocked by peroxide bridge. While for the other spin configuration, the radical centered O-atoms of peroxide bridge facilitates fully conducting channel. Thus, yielding a perfect candidate to realize a stimuli based spin filter activated by the action of light on EPO. Further, the altering degrees of conjugation in different products across the photochemical reaction pathway of EPO plays a dominant role in controlling the conductance. Molecules with fully conjugated structure yields higher conductance with strong CQI/DQI features in para/meta connections respectively, while cross conjugation results in the complete reversal of QI features with strong DQI in para connection. On the other hand, broken conjugated skeleton substantially reduces the conductance and results in complete disappearance of strong DQI dips in both para and meta connections. These generic features enables the use of EPO based device as a photoactivated molecular switch in which QI features can be toggled on and off under the action of external stimuli.

Finally, we propose another prospective of using single molecule based device for the detection of reaction radical intermediates. Like diradical intermediate of EPO, free radicals are thought to be key intermediates of various biochemical processes. Thus, the identification and isolation of free radicals possess important significance for both fundamental research and practical applications. Although wide range of analytical techniques including EPR based spectroscopy have been developed to detect the radicals. However, the recognition of short-lived radical species across a reaction pathway is extremely challenging. The present work illustrates that EPO and its related photoproducts in a single molecule junction

yields spin-unpolarized transmission. While for diradical intermediate of EPO, the spin discriminated transmission spectra governs the response of molecular device. This generic 
feature of spin polarized transmission by radical containing entity is self-explanatory and undeniable. Apart from this, one can also estimate the radicaloid character of a molecule in junction from the extent of spin polarization in the transmission spectra. Thus, we propose an idea of recognizing the radical intermediates across any reaction pathway using single molecule circuits. In this regime, scientists have already succeeded in recognition of isomers, ${ }^{49}$ DNA $^{55}$ and carbohydrates ${ }^{56}$ in single molecule junctions, however, the notion of detection of radical intermediates across a reaction pathway employing single molecule junction has not been discussed yet. Although sophisticated instruments and precise statistical data analysis algorithm is required in order to facilitate the radical intermediate detection in the single molecule conductance experiments, but the idea of pushing radical intermediate recognition capability to single molecule level is imperative to perceive and opens a new avenue to study the detailed mechanism of other pivotal chemical and biochemical processes.

\section{Acknowledgement}

Financial support from Department of Science and Technology through SERB-ECR project No. ECR/2016/000362, Indo-Sweden joint project No. DST/INT/SWD/VR/P-01/2016 is gratefully acknowledged.

Supporting Information Available: Computed MOs energetics of isolated molecules, spatial distribution of MOs of $\mathrm{B}^{*}$ in isolated phase, transmission spectra of dihydropentacene (PH), PDOS on the individual O-atoms, transmission eigenchannel analysis.

This material is available free of charge via the Internet at http:// pubs.acs.org.

\section{References}

(1) Sanvito, S. Molecular spintronics. Chem. Soc. Rev. 2011, 40, 3336-3355. 
(2) Herrmann, C.; Solomon, G. C.; Ratner, M. A. Organic radicals as spin filters. J. Am. Chem. Soc. 2010, 132, 3682-3684.

(3) Smeu, M.; DiLabio, G. A. Calculations of Electron Transport through Simple $\pi$-and $\sigma$-Type Radicals. J. Phys. Chem. C 2010, 114, 17874-17879.

(4) Bajaj, A.; Ali, M. E. Tuning of spin-polarized current in high spin organic molecules. Physica B Condens. Matter 2020, 595, 412396.

(5) Ke, G.; Duan, C.; Huang, F.; Guo, X. Electrical and spin switches in single-molecule junctions. InfoMat 2020, 2, 92-112.

(6) Gaudenzi, R.; De Bruijckere, J.; Reta, D.; Moreira, I. d. P.; Rovira, C.; Veciana, J.; Van Der Zant, H. S.; Burzurí, E. Redox-induced gating of the exchange interactions in a single organic diradical. ACS nano 2017, 11, 5879-5883.

(7) Hayakawa, R.; Karimi, M. A.; Wolf, J.; Huhn, T.; Zoprimellner, M. S.; Herrmann, C.; Scheer, E. Large magnetoresistance in single-radical molecular junctions. Nano Lett. 2016, 16, 4960-4967.

(8) Frisenda, R.; Harzmann, G. D.; Celis Gil, J. A.; Thijssen, J. M.; Mayor, M.; van der Zant, H. S. Stretching-induced conductance increase in a spin-crossover molecule. Nano Lett. 2016, 16, 4733-4737.

(9) Roldan, D.; Kaliginedi, V.; Cobo, S.; Kolivoska, V.; Bucher, C.; Hong, W.; Royal, G.; Wandlowski, T. Charge transport in photoswitchable dimethyldihydropyrene-type singlemolecule junctions. J. Am. Chem. Soc. 2013, 135, 5974-5977.

(10) Jia, C.; Migliore, A.; Xin, N.; Huang, S.; Wang, J.; Yang, Q.; Wang, S.; Chen, H.; Wang, D.; Feng, B., et al. Covalently bonded single-molecule junctions with stable and reversible photoswitched conductivity. Science 2016, 352, 1443-1445. 
(11) Ali, M. E.; Datta, S. N. Density functional theory prediction of enhanced photomagnetic properties of nitronyl nitroxide and imino nitroxide diradicals with substituded dihydropyrene couplers. J. Phys. Chem. A 2006, 110, 10525-10527.

(12) Saha, A.; Latif, I. A.; Datta, S. N. Photoswitching magnetic crossover in organic molecular systems. J. Phys. Chem. A 2011, 115, 1371-1379.

(13) Shil, S.; Misra, A. Photoinduced antiferromagnetic to ferromagnetic crossover in organic systems. J. Phys. Chem. A 2010, 114, 2022-2027.

(14) Matsumoto, I.; Ciofini, I.; Lainé, P. P.; Teki, Y. Intramolecular spin alignment within mono-oxidized and photoexcited anthracene-based $\pi$ radicals as prototypical photomagnetic molecular devices: relationships between electrochemical, photophysical, and photochemical control pathways. Chem. Eur. J. 2009, 15, 11210-11220.

(15) Suda, M.; Thathong, Y.; Promarak, V.; Kojima, H.; Nakamura, M.; Shiraogawa, T.; Ehara, M.; Yamamoto, H. M. Light-driven molecular switch for reconfigurable spin filters. Nat. Commun. 2019, 10, 1-7.

(16) Fidder, H.; Lauer, A.; Freyer, W.; Koeppe, B.; Heyne, K. Photochemistry of Anthracene9, 10-endoperoxide. J. Phys. Chem. A 2009, 113, 6289-6296.

(17) Lauer, A.; Dobryakov, A. L.; Kovalenko, S. A.; Fidder, H.; Heyne, K. Dual photochemistry of anthracene-9, 10-endoperoxide studied by femtosecond spectroscopy. Phys. Chem. Chem. Phys. 2011, 13, 8723-8732.

(18) Reddy, A. R.; Bendikov, M. Diels-Alder reaction of acenes with singlet and triplet oxygen-theoretical study of two-state reactivity. Chem. Commun. 2006, 1179-1181.

(19) Bauch, M.; Klaper, M.; Linker, T. Intermediates in the cleavage of endoperoxides. $J$. Phys. Org. Chem. 2017, 30, e3607. 
(20) Crespo-Ortiz, M. P.; Wei, M. Q. Antitumor activity of artemisinin and its derivatives: from a well-known antimalarial agent to a potential anticancer drug. J. Biomed. Biotechnol. 2012, 2012.

(21) Geroldinger, G.; Tonner, M.; Fudickar, W.; De Sarkar, S.; Dighal, A.; Monzote, L.; Staniek, K.; Linker, T.; Chatterjee, M.; Gille, L. Activation of anthracene Endoperoxides in Leishmania and impairment of mitochondrial functions. Molecules 2018, 23, 1680.

(22) Sharma, S.; Ali, M. E. Non-reductive Homolytic Scission of Endoperoxide Bond for Activation of Artemisinin: The Bi-radical Perspectives. ChemRxiv 2021, doi: https: //doi.org/10.26434/chemrxiv.14096065.v1.

(23) Martínez-Fernandez, L.; Gonzalez-Vazquez, J.; Gonzalez, L.; Corral, I. Time-resolved insight into the photosensitized generation of singlet oxygen in endoperoxides. J. Chem. Theory Comput. 2015, 11, 406-414.

(24) Neese, F. The ORCA program system. Wiley Interdiscip. Rev. Comput. Mol. Sci. 2012, 2, $73-78$.

(25) Soler, J. M.; Artacho, E.; Gale, J. D.; García, A.; Junquera, J.; Ordejón, P.; SánchezPortal, D. The SIESTA method for ab initio order-N materials simulation. J. Phys. Condens. Matter 2002, 14, 2745.

(26) Stokbro, K.; Taylor, J.; Brandbyge, M.; Ordejon, P. TranSIESTA: a spice for molecular electronics. Ann. N. Y. Acad. Sci. 2003, 1006, 212-226.

(27) Taylor, J.; Guo, H.; Wang, J. Ab initio modeling of quantum transport properties of molecular electronic devices. Phys. Rev. B 2001, 63, 245407.

(28) Datta, S. Nanoscale device modeling: the Green's function method. Superlattices Microstruct. 2000, 28, 253-278. 
(29) Troullier, N.; Martins, J. L. Efficient pseudopotentials for plane-wave calculations. Phys. Rev. B 1991, 43, 1993.

(30) Pastawski, H. M. Classical and quantum transport from generalized Landauer-Büttiker equations. Phys. Rev. B 1991, 44, 6329.

(31) Eisenthal, K.; Turro, N.; Dupuy, C.; Hrovat, D.; Langan, J.; Jenny, T.; Sitzmann, E. State-selective photochemistry of singlet oxygen precursors: kinetics and wavelength dependence of the photodissociation of anthracene endoperoxides. The Journal of Physical Chemistry 1986, 90, 5168-5173.

(32) Schmidt, R.; Schaffner, K.; Trost, W.; Brauer, H. Wavelength-dependent and dual photochemistry of the endoperoxides of anthracene and 9, 10-dimethylanthracene. The Journal of Physical Chemistry 1984, 88, 956-958.

(33) Corral, I.; González, L. Theoretical investigation of anthracene-9, 10-endoperoxide vertical singlet and triplet excitation spectra. Journal of computational chemistry 2008, 29, 1982-1991.

(34) Mollenhauer, D.; Corral, I.; González, L. Four Plus Four State Degeneracies in the OO Photolysis of Aromatic Endoperoxides. J. Phys. Chem. Lett. 2010, 1, 1036-1040.

(35) Arroyo, C. R.; Tarkuc, S.; Frisenda, R.; Seldenthuis, J. S.; Woerde, C. H.; Eelkema, R.; Grozema, F. C.; Van Der Zant, H. S. Signatures of quantum interference effects on charge transport through a single benzene ring. Angew. Chem. Int. Ed. 2013, 52, 3152-3155.

(36) Liu, J.; Huang, X.; Wang, F.; Hong, W. Quantum interference effects in charge transport through single-molecule junctions: detection, manipulation, and application. Acc. Chem. Res. 2018, 52, 151-160.

(37) Cohen, R.; Stokbro, K.; Martin, J. M.; Ratner, M. A. Charge transport in conjugated 
aromatic molecular junctions: Molecular conjugation and molecule- electrode Coupling. J. Phys. Chem. C 2007, 111, 14893-14902.

(38) Li, Z.; Smeu, M.; Afsari, S.; Xing, Y.; Ratner, M. A.; Borguet, E. Single-Molecule Sensing of Environmental pH-an STM Break Junction and NEGF-DFT Approach. Angew. Chem. Int. Ed. 2014, 53, 1098-1102.

(39) Kaliginedi, V.; Moreno-García, P.; Valkenier, H.; Hong, W.; García-Suarez, V. M.; Buiter, P.; Otten, J. L.; Hummelen, J. C.; Lambert, C. J.; Wandlowski, T. Correlations between molecular structure and single-junction conductance: a case study with oligo (phenylene-ethynylene)-type wires. J. Am. Chem. Soc. 2012, 134, 5262-5275.

(40) Lambert, C. Basic concepts of quantum interference and electron transport in singlemolecule electronics. Chem. Soc. Rev. 2015, 44, 875-888.

(41) Solomon, G. C.; Andrews, D. Q.; Hansen, T.; Goldsmith, R. H.; Wasielewski, M. R.; Van Duyne, R. P.; Ratner, M. A. Understanding quantum interference in coherent molecular conduction. J. Chem. Phys. 2008, 129, 054701.

(42) Huang, B.; Liu, X.; Yuan, Y.; Hong, Z.-W.; Zheng, J.-F.; Pei, L.-Q.; Shao, Y.; Li, J.-F.; Zhou, X.-S.; Chen, J.-Z., et al. Controlling and observing sharp-valleyed quantum interference effect in single molecular junctions. J. Am. Chem. Soc. 2018, 140, 1768517690.

(43) Alqahtani, J.; Sadeghi, H.; Sangtarash, S.; Lambert, C. J. Breakdown of curly arrow rules in anthraquinone. Angew. Chem. Int. Ed. 2018, 57, 15065-15069.

(44) Pedersen, K. G.; Borges, A.; Hedegard, P.; Solomon, G. C.; Strange, M. Illusory connection between cross-conjugation and quantum interference. J. Phys. Chem. C 2015, 119, 26919-26924. 
(45) Valkenier, H.; Guédon, C. M.; Markussen, T.; Thygesen, K. S.; van der Molen, S. J.; Hummelen, J. C. Cross-conjugation and quantum interference: a general correlation? Phys. Chem. Chem. Phys. 2014, 16, 653-662.

(46) Hansen, T.; Solomon, G. C.; Andrews, D. Q.; Ratner, M. A. Interfering pathways in benzene: An analytical treatment. J. Chem. Phys. 2009, 131, 194704.

(47) Kiguchi, M.; Nakamura, H.; Takahashi, Y.; Takahashi, T.; Ohto, T. Effect of anchoring group position on formation and conductance of a single disubstituted benzene molecule bridging Au electrodes: change of conductive molecular orbital and electron pathway. $J$. Phys. Chem. C 2010, 114, 22254-22261.

(48) Gantenbein, M.; Wang, L.; Al-Jobory, A. A.; Ismael, A. K.; Lambert, C. J.; Hong, W.; Bryce, M. R. Quantum interference and heteroaromaticity of para-and meta-linked bridged biphenyl units in single molecular conductance measurements. Sci. Rep. 2017, $7,1-9$.

(49) Zhang, Y.-P.; Chen, L.-C.; Zhang, Z.-Q.; Cao, J.-J.; Tang, C.; Liu, J.; Duan, L.-L.; Huo, Y.; Shao, X.; Hong, W., et al. Distinguishing diketopyrrolopyrrole isomers in single-molecule junctions via reversible stimuli-responsive quantum interference. $J$. Am. Chem. Soc. 2018, 140, 6531-6535.

(50) Herrmann, C.; Solomon, G. C.; Ratner, M. A. Designing organic spin filters in the coherent tunneling regime. J. Chem. Phys. 2011, 134, 224306.

(51) Donkers, R. L.; Workentin, M. S. Elucidation of the electron transfer reduction mechanism of anthracene endoperoxides. J. Am. Chem. Soc. 2004, 126, 1688-1698.

(52) Darwish, N.; Díez-Pérez, I.; Da Silva, P.; Tao, N.; Gooding, J. J.; Paddon-Row, M. N. Observation of electrochemically controlled quantum interference in a single anthraquinonebased norbornylogous bridge molecule. Angew. Chem. Int. Ed. 2012, 51, 3203-3206. 
(53) Markussen, T.; Schiötz, J.; Thygesen, K. S. Electrochemical control of quantum interference in anthraquinone-based molecular switches. J. Chem. Phys. 2010, 132, 224104.

(54) Li, X.; Tan, Z.; Huang, X.; Bai, J.; Liu, J.; Hong, W. Experimental investigation of quantum interference in charge transport through molecular architectures. J. Mater. Chem. C 2019, 7, 12790-12808.

(55) Heerema, S. J.; Dekker, C. Graphene nanodevices for DNA sequencing. Nat. Nanotechnol. 2016, 11, 127-136.

(56) Im, J.; Biswas, S.; Liu, H.; Zhao, Y.; Sen, S.; Biswas, S.; Ashcroft, B.; Borges, C.; Wang, X.; Lindsay, S., et al. Electronic single-molecule identification of carbohydrate isomers by recognition tunnelling. Nat. Commun. 2016, 7, 1-7. 


\section{Graphical TOC Entry}

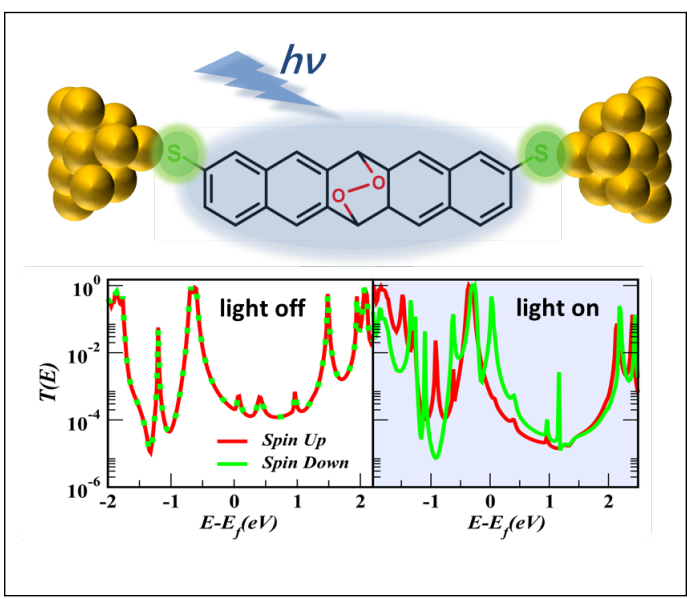

